\title{
Gestational choriocarcinoma in a postmenopausal woman. A case report and literature review
}

\author{
Tsikouras $\mathrm{P}^{1 *}$, Chatzipantelis $\mathrm{P}^{2}$, Bothou $\mathrm{A}^{1}$, Zervoudis $\mathrm{S}^{3}$, Athoulaki $\mathrm{X}^{1}$, Gaitatzi $\mathrm{F}^{1}$, Chalkidou $\mathrm{A}^{1}$, Tsirkas $\mathrm{I}^{1}$, Dragoutsos $\mathrm{G}^{1}$, Symeonidis \\ $\mathrm{P}^{1}$, Lazarou $\mathrm{A}^{1}$, Tsalkidou $\mathrm{V}^{4}$, Babageogaka $\mathrm{I}^{1}$, Peitsidis $\mathrm{P}^{3}$, Nalbanti $A T^{1}$, Alexandra Giatromanolaki $\mathrm{A}^{2}$ and Nikolettos $^{1}$ \\ ${ }^{1}$ Department of Obstetrics and Gynecology, Democritus University of Thrace, Greece \\ ${ }^{2}$ Department of Pathology, Democritus University of Thrace, Greece \\ ${ }^{3}$ Greek-French Breast Unit, Rea Hospital and University of West Attica Athens, Greece \\ ${ }^{4}$ Department of Anesthesiology, Democritus University of Thrace, Greece ${ }^{3}$ Pathology Department, National and Kapodestrian University of Athens School of \\ Medicine, Andreas Sygros Hospital, Athens, Greece
}

\begin{abstract}
Choriocarcinoma is the most common tumor of the gestational trophoblastic disease occuring usually in reproductive age related to antedecent gestational event (molar, physiological, ectopic pregnancy, elective or spontaneous abortion) either after one year or after many decades and rarely after the menopause. The main presenting clinical symptom is postmenopausal vaginal bleeding. It is an aggressive tumor with high potential rate to metastasize in various distant organs and is associated with considerable variation in prevalence wordwide.

We describe a 51-year-old postmenopausal woman, who presented with heavy vaginal bleeding 27 years after her last pregnancy, 30 years after her last abortion and 3 years after her last menstrual period. Clinical examination,vaginal ultrasound scan, and computer tomography revealed an enlarged uterus at 16 weeks pregnant Gestational choriocarcinoma's diagnosis depended on histological (preoperative endometrial curettage and postoperative uterus examination), immunochemistry findings and hCG elevated levels in serum. The intra and postoperative management was performed according to FIGO guidelines for gestational trophoblastic neoplasia.

Early diagnosis and treatment have improved the survival rate. Currently due to the rarity of the tumor in postmenopause period, the difficult differential diagnosis of non gestational choriocarcinoma is of great importance. The presentation of one more case of gestational choriocarcinoma aims to enchance the scientific experience and improve the management.
\end{abstract}

\section{Introduction}

The trophoblast is a unique tissue, with special morphological and immunohistochemical characteristics related to its various metabolic, endocrine and angiogenetic functions. Infiltration and metastasis are the main criteria for the diagnosis of a malignant tumor, but these criteria cannot be applied in the case of trophoblast, as they are normal features of this tissue, whose role is to infiltrate the endometrium, the underlying myometrium and parent vessels [1,2].

This stage is especially important in order to implant the fertilized egg, but also to feed the fetus. These biological functions are related to the morphological and immunohistochemical characteristics of this particular tissue $[3,4]$.

Trophoblast cells are an important parameter for the formation of placental tissue. Their role is to promote the penetration of the fetus inside the uterus to penetrate the uterine blood vessels, to induce angiogenesis and to produce human chorionic gonadotropin. Trophoblast is enrolled in three-layer types of cells: a) Cytotrophoblast (CT) or Langhans cells internal layer, b) Intermediate trophoblastic (IT) cells mid layer and c) and an external layer of Syncytiotrophoblastic (ST) cells $[3,4]$.

Cytotrophoblastic cells are small, round mononuclear cells with distinct cell borders, minimal clear or eosinophilic cytoplasm and vesicular nuclei. Intermediate trophoblasts are larger than cytotrophoblasts, polygonal with abundant clear cytoplasm, distinct cell borders and single nuclei. Syncytiotrophoblasts are multinucleated giant cells with eosinophilic or basophilic cytoplasm and dense, large, pyknotic nuclei. Syncytiotrophoblasts infiltrate the myometrium in order to ensure the successful implantation and proper nutrition of the fetus. This invasion process seems to be regulated mainly by the mechanism of apoptosis, which, in its turn, is controlled by the balance of two proteins belonging to the same protein family: bcl-2 protein, which demotes apoptosis and bax protein, which promotes it [5-9].

Analysis of the immunohistochemical expression of bcl-2 protein throughout pregnancy showed that this peptide is mainly expressed in the cytoplasm of the chorionic villi and extrachorionic cells of the syncytiotrophoblast. In contrast, in terms of bax protein, which promotes apoptosis, it showed a strong signal in both types of trophoblast cells

*Correspondence to: Panagiotis Tsikouras, Professor Obstetrics and Gynecology, Democritus University of Thrace, Greece, Tel: 0036974728272, E-mail: ptsikour@med.duth.gr

Key words: gestational choriocarcinoma, postmenopausal period, management

Received: August 15, 2020; Accepted: August 25, 2020; Published: August 28, 2020 
throughout pregnancy. The presence of bcl-2 protein in chorionic villi in syncytiotrophoblast cells is thought to be one of the major factors protecting these structures from premature cell death. Disorder of apoptotic regulation is likely to be associated with miscarriage [5-9]. However, persistent expression of bax protein that promotes apoptosis can contribute significantly to the balance of trophoblastic cell reproductive activity. It may also act as an antineoplastic factor [5-9].

All trophoblastic cells express the keratin coctail (pankeratin) AE1/ AE3. Additionally, syncytiotrophoblasts express hCG, hPL, and alpha inhibin [5-9].

The cytotrophoblast that covers the chorionic villi consists mainly of stem cells, while the syncytiotrophoblast is the outermost layer of trophoblast cells. It comes in contact with the mother's circulation and is responsible for the production of many hormones [10,11]. Cytotrophoblast shows intense reproductive activity, especially in the first weeks of pregnancy. This is also shown by the use of monoclonal antibodies for proliferative markers, such as PCNA and KI67 [10,11].

These markers are found in abundance in the nuclei of cytotrophoblastic cells derived from placenta of the first trimester of pregnancy, while they are rarely detected in full-term pregnancy placentas. This finding is also related to the germinal character of cytotrophoblast cells, especially during the first weeks of pregnancy. Another typical feature is the production of hCG and hPL mainly by syncytiotrophoblast $[12,13]$.

The production of these hormones varies depending on the gestational age. While hCG is produced in large amounts at the beginning of pregnancy, hPL levels peak near the end of pregnancy. These variations are due to trophoblast maturation and not only help in the correct diagnosis of trophoblastic tumors but are also excellent clinical indicators.

Choriocarcinoma and trophoblastic tumor of the placenta are the most important tumors associated with pregnancy [14]. Choriocarcinoma is an extremely aggressive tumor that originates in the trophoblast of any gestational age. Macroscopically, choriocarcinoma forms hemorrhagic polypoid masses with irregular surface,variable amounts of necrosis, dark red masses in the endometrial cavity or solid nodules infiltrating the myometrium [14-16].

Choriocarcinoma consists of cohesive sheets of trimorphic malignant trophoblasts. Cytological atypia is apparent even in low power microscopy, with numerous mitotic figures and lymphovascular invasion. Immunohistochemically, all three types of tumour cells express AE1/AE3, while ki67 is high ( $>90 \%)$. Moreover, syncytiotrophoblasts express hCG diffusely and intensely, and some intermediate trophoblasts as well, in contrast to cytotrophoblasts, which are negative for this marker [17-20]. Trophoblastic cells infiltrate blood vessels and invade the myometrium

This finding is consistent with the fact that cytotrophoblast cells are stem cells, while syncytotrophoblast cells and trophoblastic tissue intermediate cells are primarily responsible for hormone production [17-20].

Similar to pregnancy, the change from hCG production to hPL reflects and confirms the maturation of trophoblastic tissue. Based on the above-mentioned data, choriocarcinoma is considered an immature tumor with very aggressive growth and early metastases [17-20].

Placental site trophoblastic tumor is the trophoblastic tumor of the maternal placental site (Decidua basalis). This neoplasm consists mainly of intermediate trophoblastic cells without chorionic villi. Immunohistochemical staining shows a strong presence of hPL, as an indicator of a mature trophoblast. Due to this fact the placental site trophoblastic tumor is considered to be a benign tumor with expansion into the myometrium. A characteristic finding of the tumor is the presence of fibrin between cells, as well as in the perivascular spaces. Placental site trophoblastic tumour derives from mature trophoblast, as opposed to choriocarcinoma, and is considered a benign tumour with expansion into the myometrium [17-20].

From a differential point of view, the trophoblastic tumor of the placental maternal site must be distinguished from choriocarcinoma, but also from the nodules or placental maternal site plaques that are pseudo-neoplastic benign processes that also produce large amounts of hCG but also cause uterine bleeding [17-20]. These lesions are characterized by the presence of foci of intermediate trophoblastic cells inside island areas of hyaline-like material, necrotic debris of decidua [17-20].

Based on histological findings of the hysterectomy and both ovarectomy salpihngectomy, timing of the antecedent pregnancy, elective abortion and HCG titer, in order to contribute to the scientific experience and further understand gestational choriocarcinoma, we reviewed the medical record of a 51 year-old woman with gestational choriocarcinoma developing 27 years after her last pregnancy, 30 years after her last abortion and 3 years after her last menstrual period.

\section{Case presentation}

A 51-year-old Greek-Russian postmenopausal woman, gravida 8 para 4 , with 4 elective abortions, whose last period was 3 years ago visited our emergency gynecological examinations room in the Department of Gynecology and Obstetrics, Democritus University of Thrace, Greece. Her gynecological medical history included a lot of discrete episodes of irregular vaginal bleeding, associated with low abdominal pain especially in the suprapubic area, with weight loss, fatigue in the 6 months preceding her admission. She was a smoker with a 40 pack/year history spanning 25 years. Her medical history was unremarkable. No comorbidities were reported. At the time of admittance, she was in satisfactory general condition. Her gynecologic history data included unremarkable menstrual history, referred a total of four uncomplicated full-term pregnancies followed by vaginal deliveries. No postpartum complications, no data for molar pregnancy and gestational trophoblastic disease were reported. During further interview she referred, that she went through menopause at age of 48 years. She took no oral contraception's pills, or later hormone replacement therapy.

On clinical gynecological examination the uterus was palpated at approximately pregnancy 16 -week size. A vaginal sonogram confirmed a $3.2 \mathrm{~cm}$ thickened endometrial echo $8 \mathrm{~cm}$ a large tumor replace the cervix extending to the upper right vagina wall and enlarged uterus measured $8.5 \times 7.1 \times 6.2 \mathrm{~cm}$. The study patient was reassured and permanently discharged. Computer tomography imaging of pelvis abdomen, upper abdomen and chest revealed not any evidence of lymph node metastases, either in pelvis or in chest area. However, an enlarged irregulary shaped uterus containing high endometrium thickness mixed abnormal borders with blood was found. No adnexal masses were identified. Her $\beta$-hCG level was $3126.7 \mathrm{mIU} / \mathrm{ml}$, Cancer antigen 125, AFP (alpha fetoprotein) levels were normal.

Blood examination confirmed that she was anemic with a hemoglobin value of $6.7 \mathrm{~g} / \mathrm{dl}$. She received 4 blood concentrates and 3 
frozen plasma. Due to heavy metrorrhagia an endometrial biopsy was performed. Endometrial curettage consisted of blood clots, fibrinoid necrotic material, large amount of chorionic villi with hydropic degeneration and trophoblastic proliferation, along with atypical syncytiotrophoblasts, intermediate trophoblasts and cytotrophoblasts. All these features raised the possibility of complete molar pregnancy and even choriocarcinoma could not be ruled out. Furthermore, postoperative histologic diagnosis was suggested for determining the presence of choriocarcinoma

The levels of $\beta$-hCG postoperatively were $5575 \mathrm{mIU} / \mathrm{ml}$ and $5689 \mathrm{mIU} / \mathrm{ml}$ and the vaginal bleeding continued. The mentioned woman underwent 3 weeks after the fractional curettage in abdominal hysterectomy and bilateral salpingo-oophorectomy.

According to the World Health Organisation FIGO prognostic score for gestational trophoblastic disease based on investigative findings, our patient had a score of 6 making her low risk for resistance to monotherapy and classified her as FIGO stage I gestational trophoblastic neoplasia. Histologic examination revealed largely necrotic material as follows: Representative sections consisted of sheets of trimorphic malignant cells (cytotrophoblasts, intermediate trophoblasts, syncytiotrophoblasts) (Figures 1A-1C). The cells had large irregular nuclei, with visible nucleoli and eosinophilic or clear cytoplasm. Lymphovascular spaces with fibrin necrosis and neoplastic emboli were also noted. On immunohistochemistry, neoplastic cells were positive for AE1/AE3, ki67 and hCG (Figures 2A-2C). Cancer antigen CA125 and AFP levels were within normal range.

The postoperative course was uncomplicated, the patient was clinically asymptomatic, and attended on regular follow up check program in our Oncology Department. The serum $\beta$-hCG titer fell gradually until normalization of serum $\beta$-hCG values. All follow up examinations revealed no pathological findings. Monotherapy of methotrexate has been administered to protect the patient's health and reduce the risk of disease relapse.

\section{Discussion}

Trophoblast disease of pregnancy or gestational trophoblastic disease includes a unique tissue group with a wide range of endocrine and angiogenic functions derived from placental trophoblasts. They are associated with uncommon, interrelated conditions, which differ according to the following parameters: invasion, regression, metastasis and recurrence rate [21-24]. Gestational trophoblastic disease is the appropiate collective name but interrelated pathological conditions derived from placental trophoblasts for partial, complete molar pregnancy (both characterized by the presence of hydropic villi which usally contain fetal vessels and fetal red blood cells), miscallaneous trophoblastic tumors (exaggerated placental site, placental site nodule or plaque), unclassified trophoblastic lesions, choriocarcinoma and placental site trophoblastic tumor [21-24].

Malignant forms of trophoblastic cells, both those resulting from mole pregnancy and those resulting from malignant mutation of healthy placenta cells, share many of these characteristics. Trophoblast cells inverse the uterus layers, promote the angiogenesis, malignant cells are also able to make distinct metastases. The hCG production is always maintained, helps to establish diagnosis and monitoring the response to treatment [21-24].

The mentioned subgroups of gestational trophoblastic diseases (including hydatide mole partial or complete invasive mole
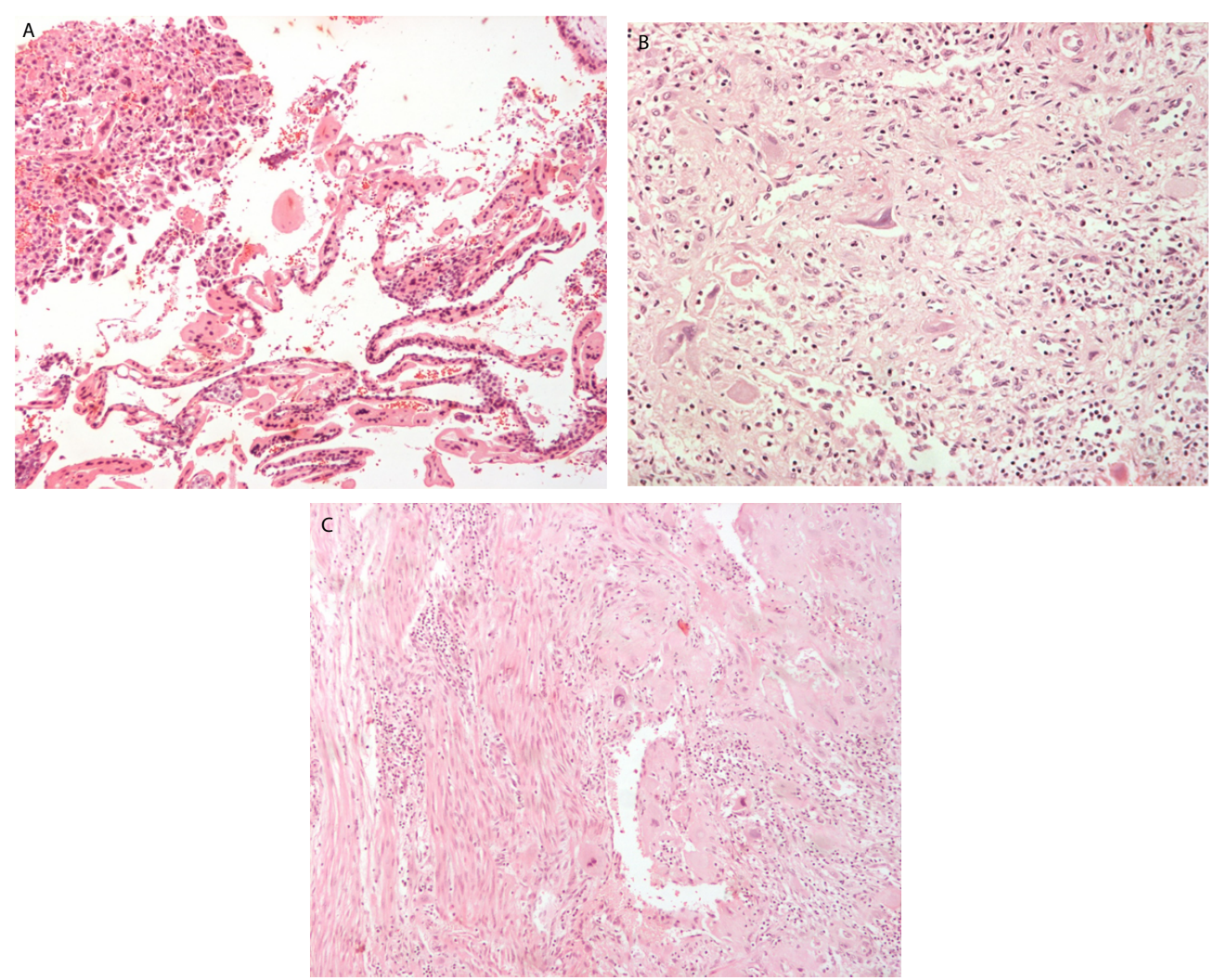

Figure 1. (A) Atypical trophoblastic cells in curettage specimen x10 (H\&E) (B) Syncytiotrophoblasts and intermediate trophobasts x 20 (H\&E) (C) Atypical trophoblastic cells invading myometrium x 10 (H\&E) 


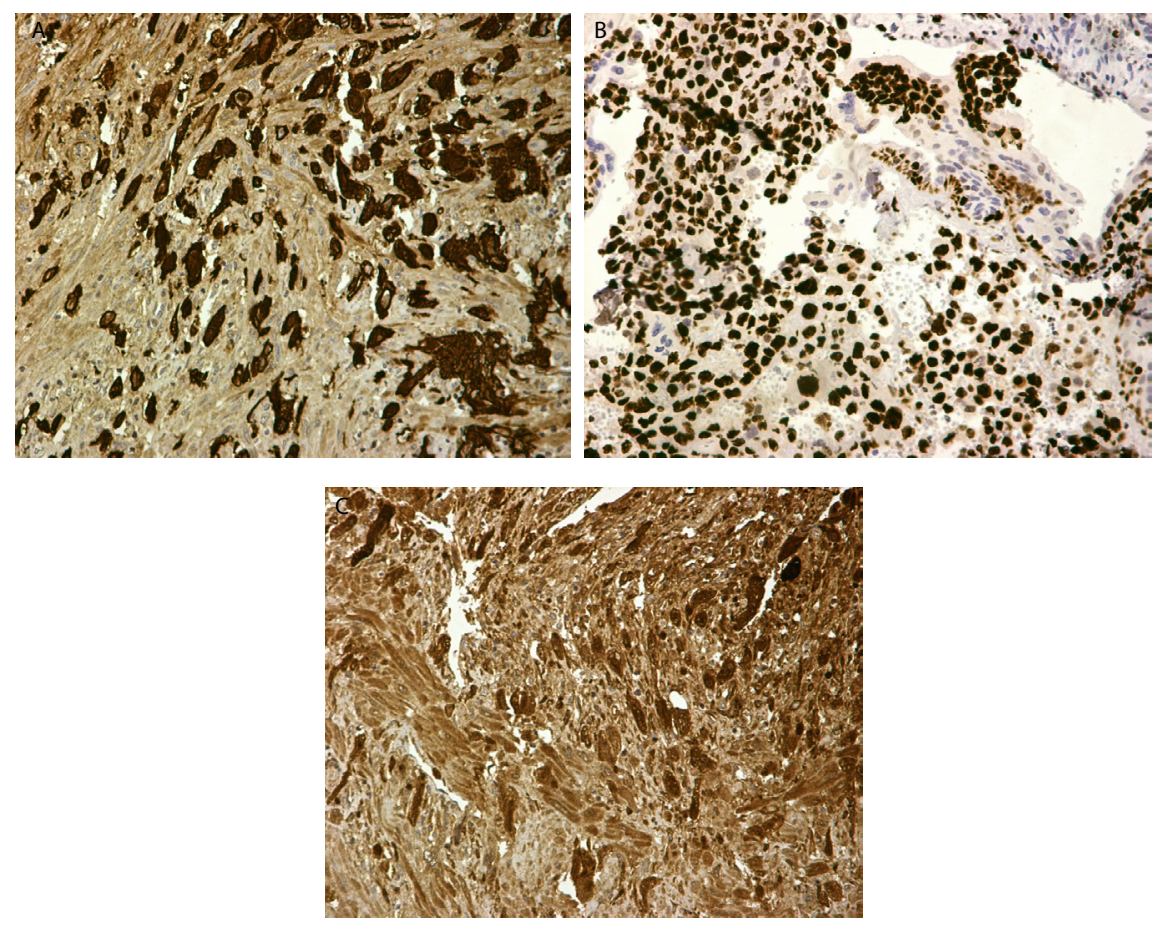

Figure 2. (A) Cytokeratin AE1 AE3 positive cells (B) Ki 67 stain over $90 \%$ of tumour cells (C) Stain for bhCG X 20

choriocarcinoma and tumor of placenta insertion site) differ in possibility for invasion, metastasis, recurrence and total regression. Trophoblastic diseases can also occur independent to gestation (non gestational chorioncarcinomas), are biphasic proliferation of cytotrophoblast and syncytiotrophoblast (hormone secreting component) with anatomical morphological structure similarities to trophoblast tissue of placental previllous stage but without chorionic villi. Concerning the frequency of appearance, the choriocarcinoma is in the majority of cases gestational, primarily intrauterine located, in cases of extrauterine gestational arising at position of ectopic pregnancy and in cases of non gestational possibly derived from pluripotent germ cells mostly in the ovaries high malignant variant. Primary non gestational choriocarcinoma derives from ovary germ cell tumors, residual germ cells or dedifferentiation of endometroid adenocarcinoma and referred mainly to anatomical locations in ovaries and rarely in uterus in postmenopausal period [2528].

Gestational chorioncarcinoma can occur by any gestational event as follows: after hydatidiform mole in $50 \%$ of cases, $25 \%$ after spontaneous abortion, $22.5 \%$ after normal pregnancy and $2.5 \%$ after ectopic pregnancy ( 1 in 5333 tubal pregnancies). Not always to the predisponding pregnancy within the uterine cavity is referred as one of the most related factors within a year of the referred pregnancy. Especially in the premenopausal women gestational trophoblastic pathology is subsequent hydatid mole in $60 \%$ of cases, abortions in $30 \%$ and in $10 \%$ normal or ectopic pregnancy. While in Europe and North America the occurrence is 30.000-40.000 intrauterine and 1/40 molar pregnancies, the rates increase to $1 / 500-3000$ in south east Asia. The exact incidence in postmenopausal period is extremely low, as satisfactory data to estimate are not available. Only a few cases since 1970 have been reported which had occurred after a long latent time from last pregnancy. The Choriocarcinomas, which are highly vascular neoplasms have as main clinical presentation abnormal uterine bleeding and matestasize readily in approximately $30 \%$ of cases at the time of the final diagnosis and most commonly in lung $60-75 \%$, vagina $40-50 \%$, brain $15-20 \%$, liver $15-20 \%$, spleen $10 \%$, intestines $10 \%$ (5\% bowel metastasis), and central nervous system involvement in $10 \%$ of cases, very rare manifestation, $4 \%$ in cardiac intracavity mass, cardiac metastasis. Approximately $0.76-4 \%$ of choriocarcinomas referred to occur in ectopic anatomic locations and they are associated with more aggressive character with distant metastasis [25-31].

Additionally, to these references there were following complications and metastasis locations, specifically: Hcg influence in the thyreotropic action to thyreotoxis based on similar molecular structure Hcg and TSH thyroid stimulating hormone. Hypercoagulability (increasing the activation of platelets, factor XII, X, cytokines TNFa, IL-1,IL-6) [25-31].

Other uncommon sites of chorioncarcinoma metastasis and very rare locations are as follows: in skin 1.4-5\%, choroid, uvea, maxillary sinus, intranasal area and also in solid organ transplants, like kindey and liver [14,32-35].

Gestational trophoblastic neoplasia occurs most commonly in reproductive age after antedecent gestational events, like recent pregnancy usually within a year of a preceding pregnancy or more decades in the past, molar or non-pregnancy in $50 \%$ of the cases, abortions, ectopic pregnancies also recently or after a long time in the past, while only a few cases have been reported in the international literature. In complete molar pregnancies the classical occurrence of large edematosus clusters of vesicles with hemorrhage and necrosis is typical [14,32-35]. The majority karyotype of complete molar pregnancies are 46XX in 95\% of cases arise arising from fertilization of an empty pronucleus by haploid sperm that undergoes duplication, Diandric diploidy, while the minority are $46 \mathrm{XY}$ in 5\% of cases which arise from fertilization of an empty egg by two sperm and led to Diandric dispermy. Partial mole is associated to less abundant hydropic villi large edematous with stromal fibrosis [14,36-38]. Typically, the karyotype in partial mole is triploidy which results from fertilization of oocyte 
by either duplicated spermatozoa, or two spermatozoa respectively 69 chromosomes 69XXY 70\% monogynic, 69XXX 27\% diandric 69XYY in $3 \%$. Recurrence of complete and partial mole pregnancy rates are $2-4 \%$ and $1.8 \%$ respectively $[14,36-38]$.

The expressions of these proteins (bcl-2 protein, bax protein) in cases of gestational trophoblastic disease, together with DNA analysis are very helpful tools regarding the characterization of each tumour [14,36-38].

Gestational Choriocarcinoma is considered a relatively rare tumour that is curable in most cases, with an excellent prognosis regarding future fertility. The mentioned tumor has been referred to as the most common malignant gestational trophoblastic tumor and occurs preferentially in women of reproductive age. Placental site trophoblastic tumour derives from mature trophoblast, as opposed to choriocarcinoma, and is considered a benign tumour with expansion into the myometrium [36-38].

DNA analysis can help to determine which type of gestational event had appeared recently or further into the past and suggest gestational trophoblastic neoplasia. Tumors with paternal only chromosomes originate rather from a complete hydatid-shaped mole pregnancy. In contrast, tumors which include both maternal and paternal chromosomes are a complication of a physiological pregnancy, miscarriage, or ectopic pregnancy. This can also be seen using the method of restriction fragments length polymorphism or short tandem repeat (STR) genotyping using locus specific VNTR (variable number of tandem repeats, makro and minisatellites). The diploid appearance of the complete hydatid-mole pregnancy can be detected using the FISH method and with a DNA analysis against a specific chromosome, in which we can find two signals [39-42].

One mechanism that could explain the malignant differentiation of trophoblast cells in the complete hydatid mole membrane is the identification of the expression of the c-myc oncogene, which is located on chromosome 8q24. This is responsible for the production of a phosphoprotein that acts as a transcription factor [39-42].

It is of great clinical importance to early recognize the type of choriocarcinoma because the prognosis and treatment of the two subtypes is different. The difference between the two main types of gestational and non-gestational choriocarcinoma based on the previously occurring gestational event (absolute majority is gestational). In cases of absence of gestation in the past and the nongestational variant is difficult to diagnose except in women, who are sexually immature or with conception problems [43-46].

There are no clear ultrasound and immunohistohstochemical diagnostic criteria to definitively identify the choriocarcinoma subtype.

DNA polymorphism analysis based on the woman's and her partner's blood, tumor tissue and human leukocyte antigen typing for antigens of paternal origin in trophoblastic tissue can possibly lead to early identification of the choriocarcinoma subtype and start treatment. The cited method has an advantage to identify the choriocarcinoma subtype as in cases of gestational revealed paternal and maternal alleles in same amounts in tumor cells while in nongestational cases only maternal allele are found [39-42].

According to published literature, in cases of nongestasional choriocarcinoma no standard of treatment exists, generally perfomed like as in gestational choriocarcinima with resistance to chemotherapy and worse survival rate.
In our case, the indication of confirmation gestational choriocarcinoma due to DNA analysis test non availability was based on high $\beta$-hCG serum levels, histological evident findings comprising of malignant cytotrophoblast, syncytiotrophoblast and indermediate trophoblast cells without chorionic villi and support of immunohistochemistry tests for $\beta$-hCG, a-inhibin and Cytoceratins. Due to low risk score and early stage according to FIGO staging we recommended monochemotheraphy, based on international oncology treatment protocols. This reported case is of clinical importance due to the patient's long 30-year period after her last gestational event age of patient 3 years postmenopausal long time after last gestational event. Generally, the prognostic value in reproductive time of the long duration between last gestation event and tumor diagnosis is negative associated to poor prognosis, however in the postmenopausal time unknown, possible poor. The diagnosis was based on histological, immunochemistry and $\beta$-hCG findings in serum.

\section{Conclusion}

Gestational trophoblastic disease is generally considered to be a curable disease, but the close cooperation of the Gynecologist, the Pathologist and the Oncologist is a requirement towards that end. More future multicentric research studies are necessary to improve the quality of early diagnosis and management for this rare disease.

\section{References}

1. Ngan HY, Kohorn EI, Cole LA, Kurman RJ, Kim SJ (2012) Trophoblastic disease. Int J Gynaecol Obstet 2: 130-136.

2. Allias F, Bolze PA, Gaillot-Durand L (2014) Gestational trophoblastic disease. Ann Pathol 34: 434-447.

3. Braga A, Mora P, de Melo AC, Nogueira-Rodrigues A, Amim-Junior J, et al. (2019) Challenges in the diagnosis and treatment of gestational trophoblastic neoplasia worldwide. World J ClinOncol 10: 28-37.

4. Ning F, Hou H, Morse AN, Lash GE (2019) Understanding and management of gestational trophoblastic disease. F1000Res 10: 8.

5. Niemann I, Vejerslev LO, Frøding L, Blaakær J, Maroun LL, et al. (2015) Dan Gestational trophoblastic diseases - clinical guidelines for diagnosis, treatment, followup, and counselling. Med J 62: 2.

6. Seckl MJ, Sebire NJ, Berkowitz RS (2010) Gestational trophoblastic disease. Lancet 28: 717-729.

7. Soper JT, Mutch DG, Schink JC (2004) Diagnosis and treatment of gestational trophoblastic disease: ACOG Practice Bulletin No. 53. American College of Obstetricians and Gynecologists. GynecolOncol 93: 575-585.

8. Committee on Practice Bulletins-Gynecology (2004) ACOG Practice Bulletin \#53. Diagnosis and treatment of gestational trophoblastic disease. Obstet Gynecol 103: 1365-1377. [Crossref]

9. Kohorn E (2004) Practice bulletin No. 53--Diagnosis and treatment of gestational trophoblastic disease. Obstet Gynecol 104: 1422

10. Brown J, Naumann RW, Seckl MJ, Schink J (2017) 15years of progress in gestational trophoblastic disease: Scoring, standardization, and salvage. GynecolOncol 144: 200207.

11. Goldstein DP, Berkowitz RS (2011) Current management of gestational trophoblastic neoplasia. HematolOncolClin North Am 26: 111-131.

12. Gueye M, Kane-Gueye SM, Ndiaye-Gueye MD, Mbaye M, Diouf AA (2014) Moreau JCGestational trophoblastic neoplasia after achieving a nondetectable serum human chorionic gonadotrophin level. BJOG 121: 1415-1419.

13. Froeling FE, SecklMJ.Cole LA, Sutton JM (2003) HCG tests in the management of gestational trophoblastic diseases. ClinObstet Gynecol 46: 523-540.

14. Smith HO, Kohorn E, Cole LA (2005) Choriocarcinoma and gestational trophoblastic disease. ObstetGynecolClin North Am 32: 661-684.

15. Cole LA, Khanlian SA, Giddings A, Butler SA, Muller CY (2006) Gestational trophoblastic diseases: 4. Presentation with persistent low positive human chorionic gonadotropin test results. GynecolOncol 102: 165-172. 
16. Soper JT (2006) Gestational trophoblastic disease. Obstet Gynecol 108: 176-187.

17. Diver E, May T, Vargas R, Bernstein M, Goldstein D (2013) Changes in clinical presentation of posttermchoriocarcinoma at the New England Trophoblastic Disease Center in recent years. GynecolOncol 130: 483-486.

18. Macdonald MC, Ram R, Tidy JA, Hancock BW (2010) Choriocarcinoma after a nonterm. J Reprod Med. 55: 213-218.

19. Song L, Li Q, Yin R, Wang D (2018) Choriocarcinoma with brain metastasis after A case report. Medicine 97: e12904.

20. Rodabaugh KJ, Bernstein MR, Goldstein DP, Berkowitz RS (1998) Natural history of posttermchoriocarcinoma. J Reprod Med 43: 75-80.

21. Ngan HY, Bender H,Benedet JL, Jones H, Montruccoli GC, Pecorelli S (2003) Gestational trophoblastic neoplasia, FIGO Committee on Gynecologic Oncology. Int J Gynaecol Obstet 1: 175-177.

22. Benedet JL, Bender H, Jones H, Ngan HY, Pecorelli S (2000) FIGO staging classifications and clinical practice guidelines in the management of gynecologic cancers. Int J Gynaecol Obstet 70: 209-262.

23. Kohorn EI (2007) Dynamic staging and risk factor scoring for gestational trophoblastic disease. J Int J Gynecol Cancer 17: 1124-1130.

24. Kohorn EI (2007) Negotiating a staging and risk factor scoring system for gestational trophoblastic neoplasia. A progress report. J Reprod Med 47: 445-450.

25. Wang L, Wan Y, Sun Y, Zhang X, Cheng X (2019) Cancer Biol Pure nongestational uterine choriocarcinoma in postmenopausal women: a case report with literature review. Ther 20: 1176-1182.

26. Kaabia O, Meddeb S, Rhim MS, Bibi M, Khairi H (2014) The uterine choriocarcinoma in postmenopausal women: specificities of diagnosis and treatment. Pan Afr Med J 19: 176 .

27. U Mukherjee, V Thakur, D Katiyar, Hari K Goyal, D Pendharkar (2006) Uterine choriocarcinoma in a postmenopausal woman. Med Oncol 23: 301-303.

28. Georges El Hasbani, AlaaBalaghi, Kamal Tarabine, Richard Assaker, Michel Samaha, HadiGhanem (2018) BasselAbouzeid Uterine choriocarcinoma diagnosed 11 years after menopause: A case report Case Rep Womens Health 20: $\mathrm{e} 00076$.

29. Monica Garcia, Rita L Romaguera, Carmen Gomez-Fernandez (2004) A hydatidiform mole in a postmenopausal woman. A case report and review of the literature. Arch Pathol Lab Med 128: 1039-1042.

30. El-Agwany AS, Rady HA, Hegab HM (2017) Complete molar pregnancy in postmenopausal woman. Indian J SurgOncol 8: 185-187.

31. Tsukamoto N, Iwasaka T, Kashimura Y, Uchino H, Kashimura M (1985) Gestational trophoblastic disease in women aged 50 or more. GynecolOncol 20: 53-61.
32. Karadeniz T, Topsakal M, Ozkaptan O, Cakır C (2011) Bilateral renal choriocarcinoma in a postmenopausal woman. Korean J Urol 52: 498-501.

33. Mangla M, Singla D, Kaur H, Sharma S (2017) Taiwan Unusual clinical presentation of choriocarcinoma: A systematic review of case reports. J Obstet Gynecol 56: 1-8.

34. Dombrovsky I, Tilden HR, Aftandilians T, Wong S, Stowe RJ (2020) Metastatic brain choriocarcinoma in a postmenopausal woman: A Case Report. Am J Case Rep 21 : e917656.

35. Massenkeil G, Crombach G, Dominik S, De Bruyne F, Nitz U (1996) Metastatic choriocarcinoma in a postmenopausal woman. GynecolOncol 61: 432-437.

36. Na Guo, Rutie Yin, Qingli Li, Liang Song, Danqing Wang (2018) Postmenopausa choriocarcinoma: a rare case report and review of the literature. Menopause 25: 239241.

37. Xiaofei Zhang, Caiyun Zhou, Minghua Yu, Xiaoduan Chen (2015) Coexisting epithelioid trophoblastic tumor and placental site trophoblastic tumor of the uterus following a term pregnancy: report of a case and review of literature. Int $J$ ClinExpPathol 8: 7254-7259.

38. Park SH, Park A, Kim JY, Kwon JH, Koh SB (2009) A case of non-gestational choriocarcinoma arising in the ovary of a postmenopausal woman. J GynecolOncol 20: 192-194.

39. Wang Y, Yang Y, Teng F, Zhang H, Xue F (2014) Pure nongestational uterine choriocarcinoma in a postmenopausal Chinese woman confirmed with short tandem repeat analysis. Am J Obstet Gynecol. 211: e1-3.

40. O'Neill CJ, Houghton F, Clarke J, McCluggage WG (2008) Uterine gestationa choriocarcinoma developing after a long latent period in a postmenopausal woman: the value of DNA polymorphism studies. Int J SurgPathol 16: 226-229.

41. Hirata Y, Yanaihara N, Yanagida S, Fukui K, Iwadate K (2012) Molecular genetic analysis of nongestationalchoriocarcinoma in a postmenopausal woman: a case report and literature review. Int J GynecolPathol 31: 364-368.

42. LazareC, ZhiW, DaiJ, CaoC, SookhaRR (2019) A pilot study comparing the genetic molecular biology of gestational and non-gestational choriocarcinoma. Am J Transl Res 11: 7049-7062.

43. Chittenden B, Ahamed E, MaheshwariA (2009) Choriocarcinoma in a postmenopausal woman. Obstet Gynecol 114: 462-465.

44. Desai NR, Gupta S, Said R, Desai P, Dai Q (2010) Choriocarcinoma in a 73-year-old woman: a case report and review of the literature. J Med Case Rep 4: 379.

45. Yousefi Z, Mottaghi M, Rezaei A, Ghasemian S (2016) Abnormal presentation of choriocarcinoma and literature review. Iran J Cancer Prev 24: e4389.

46. Samal SK (2014) Postmenopausal choriocarcinoma: A rare case report. J Midlife Health 5: 159-161. [Crossref]

Copyright: (C2020 Tsikouras P. This is an open-access article distributed under the terms of the Creative Commons Attribution License, which permits unrestricted use, distribution, and reproduction in any medium, provided the original author and source are credited. 